\title{
Hysteresis Effect in Mean-Field Model
}

\author{
R. PEŁKA*
}

The H. Niewodniczański Institute of Nuclear Physics Polish Academy of Sciences

Radzikowskiego 152, 31-342 Kraków, Poland

\begin{abstract}
A simple feedback loop and a mean-field approximation are employed to simulate the memory effect in the exchange-coupled magnetic system. The nonlinearity of the feedback turns out to be the crucial factor for the appearance of hysteresis loops. A threshold value above which the hysteresis disappears is the mean-field transition temperature. Temperature dependence of the coercive field is investigated.
\end{abstract}

DOI: $10.12693 /$ APhysPolA.126.28

PACS: 75.60.-d, 75.50.Vv, 75.60.Ej

\section{Introduction}

The phenomenon of hysteresis is omnipresent in the world of physical systems. It was introduced into scientific vocabulary about 130 years ago by the Scottish physicist Alfred Ewing [1]. Hysteresis occurs in many engineering, physical and economic systems. It has been established that the prerequisite feature for it to appear is the nonlinearity of the underlying dynamics. In the area of magnetic phenomena, the early phenomenological model of Lord Rayleigh formed the basis of the wellknown Preisach model of ferromagnetic hysteresis, which has been further developed and widely discussed in a long series of papers appearing up to the present day [2-4]. Stoner and Wohlfarth [5] explained the lag of the magnetization behind the external magnetic field within a simple micromagnetic model for the case of a single domain particle of uniform magnetization. In their approach, the crucial role was played by the magnetic anisotropy. The advances in the statistical and computational physics enabled the investigation of hysteretic phenomena in realistic multiparticle and multidomain systems. The most important results on hysteresis modeling are reviewed in a number of useful monographs published during the nineties of the last century $[3,6,7]$. The model considered here is a quasi-classical model of quantum spins interacting via a mean-field. It is demonstrated that a simple feedback loop in such a system leads to the occurrence of the memory effect. Most interestingly there is no internal anisotropy term in the model system, the spin-spin interaction being of the isotropic Heisenberg type. The only source of anisotropy is the interaction with the external magnetic field. The laws of statistical physics averaging out the contributions from the field-split multiplets introduce to the system the nonlinearity that turns out sufficient to yield the nontrivial lag of the magnetization behind the changing external magnetic field.

\section{Results}

Let us briefly describe the model components. The defining feature of the system is its Hamiltonian, which in the present case contains the isotropic Heisenberg coupling

*e-mail: robert.pelka@ifj.edu.pl

$$
\hat{H}=-J \sum_{<i j>} \hat{S}_{i} \cdot \hat{S}_{j}+g \mu_{B} \sum_{i} \hat{S}_{i} \cdot \mathbf{H}_{e x t}
$$

where $\langle i j\rangle$ denotes the summation over the nearestneighbor pairs of spins and $J$ is the exchange coupling constant. The second term is the Zeeman interaction with the external magnetic field, where $g$ is the spectroscopic factor and $\mu_{B}$ denotes the Bohr magneton. In the mean-field approximation, the interaction pattern dependent on the lattice topology is simplified by the replacement of the interaction with the neighboring spins by that with the statistical expectation value of a single spin operator. In this approximation the Hamiltonian may be rewritten as follows

$$
\hat{H}=g \mu_{B} \hat{S} \cdot\left(\mathbf{H}_{e x t}+\mathbf{H}_{M F}\right)
$$

with $H_{M F}=-z J<\hat{S}>/\left(g \mu_{B}\right)$, where $z$ denotes the number of the nearest neighbours. On carrying out the standard statistical averages one arrives at the formula for the mean value of the magnetic moment which can be readily translated in terms of the mean-field.

$$
H_{M F}=\frac{z J S}{g \mu_{B}} B_{S}\left[\frac{\mu_{B} g S\left(H_{e x t}+H_{M F}\right)}{k_{B} T}\right]
$$

where $S$ is the spin quantum number, and $B_{S}$ denotes the Brillouin function. Equation 3 is the core of our considerations. It defines a simple feedback loop, which yields the saturated value of the mean magnetization $<\hat{\mu}>=\left(g \mu_{B}\right)^{2} /(z J) H_{M F}$ if a sufficiently high number $N$ of recursions is performed.

In what follows, the reduced measure of temperature is used $t=T / T_{c}$ where $T_{c}=z J S(S+1) /\left(3 k_{B}\right)$ is the mean-field critical temperature. The magnetization is normalized with respect to the maximal possible value per spin, i.e., $\mu_{\max }=g \mu_{B} S$. The external magnetic field $H_{\text {ext }}$ is measured relative to the maximal value of the coercive field $H_{c \max }=z J S /\left(g \mu_{B}\right)$. Similarly, a dimensionless unit is introduced to measure the hysteresis area in the magnetization versus external magnetic field plane (denoted here by $S_{h}$ ) by reference to its maximal possible value $S_{h \max }=4 \mu_{\max } H_{c \max }$. In the dimensionless units $t, h_{M F}=H_{M F} / H_{c \max }, h=H_{\text {ext }} / H_{c \max }$, Eq. (3) reads

$$
h_{M F}=B_{S}\left[\frac{3 S}{S+1} \frac{h+h_{M F}}{t}\right]
$$

As can be seen from Eq. (4) the description of the evolution of the loops in terms of the reduced quantities 
is independent of the specific value of the coupling constant $J$, spectroscopic factor $g$, and the nearest neighbor number $z$, which all enter the energy scale given by $T_{c}$. However, the formula implies a dependence on the spin value $S$. Figure 1 shows the temperature dependence of hysteresis loops for two values of spin quantum number $S=1 / 2$ and $S=2$, and for $N=100$. One can see that at very low temperatures the hysteresis loop acquires the limiting shape of a rectangle. On raising the temperature it shrinks and vanishes above the transition temperature $t_{c}=1$. For higher spin values the coercive fields are suppressed which is consistent with the fact that the interaction increases with increasing spin value.

Figure 1 depicts also the temperature dependence of the hysteresis area for $S=1 / 2$ and $N=100$. The hysteresis area monotonously decreases with increasing temperature vanishing at $t_{c}=1$. In the case of a high number of recursions, the coercive field can be found numerically.
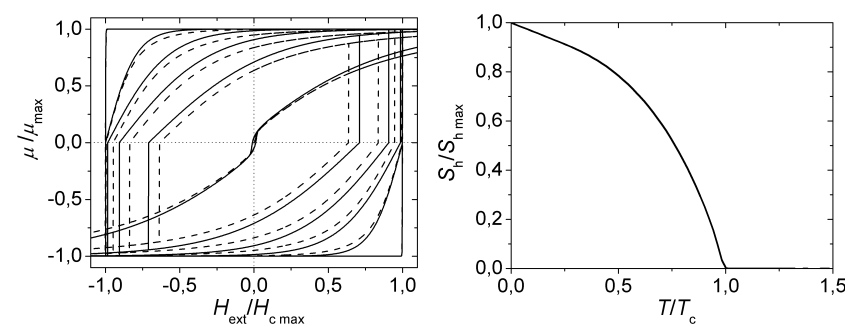

Fig. 1. Left: Hysteresis loops obtained for $S=1 / 2$ (solid line) and $S=2$ (dashed line) with $N=100$ at temperatures $t=0.002,0.2,0.4,0.6,0.8,1.0$. Right: Temperature dependence of the hysteresis area for $S=1 / 2$.
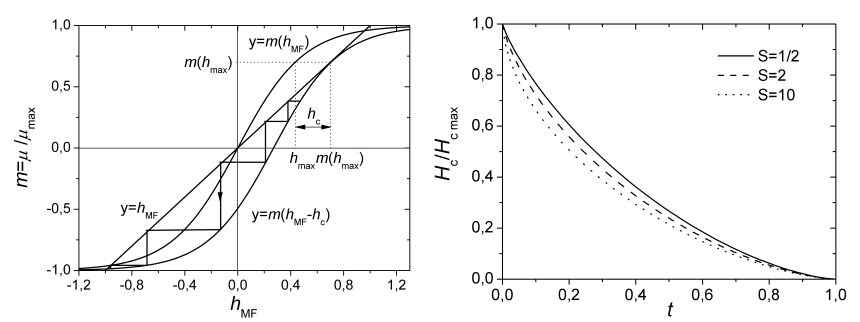

Fig. 2. Left: Configuration of the magnetization curve corresponding to the external field being equal to coercive field. Right: Temperature dependence of the coercive field.

Figure 2 shows two magnetization curves as functions of the mean field $m\left(h_{M F}\right)=<\hat{\mu}>/ \mu_{\max }=$ $B_{S}\left[3 S h_{M F} /(t(S+1))\right]$. One curve goes through the origin of the coordinate frame and corresponds to the case where the external magnetic field vanishes. The other curve is shifted to the right so that the line $y=h_{M F}$ is tangent to it. As the zigzag curve, illustrating successive moves of the feedback loop indicates, the latter configuration corresponds to the threshold at which the magnetization will flip over (change sign). The corresponding shift can be found as the value of the following function
$f\left(h_{M F}\right)=m\left(h_{M F}\right)-h_{M F}$ at its maximum $h_{\max }$. Indeed, the necessary condition for the maximum ensures the relevant curves are tangent. In Fig. 2 is also demonstrated that the value of the function at its maximum is equal to the shift of the magnetization curve and hence to the coercive field. Figure 2 (right) shows the coercive field vs. reduced temperature for three indicated spin number values. There is a rather weak $S$-dependence, practically not changing for $S>10$.

\section{Conclusions}

Firstly, it is quite surprising that such a simple feedback loop based on the principles of statistical physics should display a hysteretic behavior. This fact enforces the view that one of the key conditions that must be satisfied for the hysteresis loop to appear is the nonlinearity of the underlying model. Another crucial prerequisite is the state of broken symmetry, which here is realized by the action of the external magnetic field. The shape of the loops were shown to depend on the spin number value $S$, which intuitively derives from the $S$-dependence of the interaction energy with external magnetic field. At the same time, it remains independent of the magnitude of exchange interaction $J$ and the coordination number $z$ defining the internal energy scale in the system. The threshold above which the hysteresis loop disappears was shown to be placed at the transition temperature $T_{c}$. The scale of the coercive field defined by its maximal value $H_{c \max }$ is proportional to the ratio of the internal energy scale $z J S^{2}$ to the single site magnetic moment $g \mu_{B} S$. Therefore, in contrast to the StonerWohlfarth model the relevant quantity in the mean-field model is not anisotropy but isotropic exchange interaction. The fact that the loops were obtained within a physically plausible model points to the fact that they may be treated as basic ingredients of the well-known Preisach model, i.e., hysterons. Moreover, a less rough treatment of spin interactions, where one would take duly into account the quantum spin correlations, will probably give the scheme sensitive to the coordination number $z$, i.e. to the topology of the spin lattice.

\section{References}

[1] J.A. Ewing, Philos. Trans. R. Soc. London 176, 523 (1885).

[2] Lord Rayleigh, Philos. Mag. 23, 225 (1887); F. Preisach, Z. Phys. A 94, 277 (1935).

[3] A. Visintin, Differential Models of Hysteresis Springer-Verlag, Berlin, 1994.

[4] G. Shabo, G. Kadar, Phys. Rev. B 58, 5584 (1998); G. Kadar, Physica B 275, 40 (2000); G. Shabo, G. Kadar, Physica B 275, 187 (2000); G. Kadar, G. Shabo, J. Magn. Magn. Mat. 215-216, 592 (2000).

[5] E.C. Stoner, E.P. Wohlfarth, Philos. Trans. R. Soc, London, Ser. A 240, 599 (1948).

[6] I.D. Mayergoyz, Mathematical Models of Hysteresis Springer-Verlag, New-York, 1991.

[7] G. Bertotti, Hysteresis in Magnetism Academic Press, San Diego, 1998. 